Published in final edited form as:

Subcell Biochem. 2007 ; 43: 77-98.

\title{
Synaptosome Proteomics
}

\author{
Fengju Bai ${ }^{1}$ and Frank A. Witzmann ${ }^{2}$ \\ ${ }^{1}$ Safety Sciences, Charles River Laboratories Preclinical Services, USA \\ ${ }^{2}$ Indiana University School of Medicine Biotechnology Research \& Training Center, USA
}

\begin{abstract}
Our knowledge of the complex synaptic proteome and its relationship to physiological or pathological conditions is rapidly expanding. This has been greatly accelerated by the application of various evolving proteomic techniques, enabling more efficient protein resolution, more accurate protein identification, and more comprehensive characterization of proteins undergoing quantitative and qualitative changes. More recently, the combination of the classical subcellular fractionation techniques for the isolation of synaptosomes from the brain with the various proteomic analyses has facilitated this effort. This has resulted from the enrichment of many low abundant proteins comprising the fundamental structure and molecular machinery of brain neurotransmission and neuroplasticity. The analysis of various subproteomes obtained from the synapse, such as synaptic vesicles, synaptic membranes, presynaptic particles, synaptodendrosomes, and postsynaptic densities (PSD) holds great promise for improving our understanding of the temporal and spatial processes that coordinate synaptic proteins in closely related complexes under both normal and diseased states. This chapter will summarize a selection of recent studies that have drawn upon established and emerging proteomic technologies, along with fractionation techniques that are essential to the isolation and analysis of specific synaptic components, in an effort to understand the complexity and plasticity of the synapse proteome.
\end{abstract}

\section{Introduction}

Neuroscience is a discipline in which proteomics is having a growing impact. Many neuropsychiatric and neurodegenerative diseases, such as Alzheimer's, are thought to involve altered expression of multiple structural and/or metabolic genes and proteins, and therefore are well-suited for proteomic analysis (Kim et al. 2004). The study of other conditions, such as addiction and mood disorders that likely are secondary to altered expression of proteins involved in neurotransmission or neuroplasticity, can also take advantage of the power of global and narrow protein profiling that proteomics offers, for example, to examine the role of synaptic proteins in different disease states.

However, when using proteomics to study central nervous system (CNS) function and pathology, one is faced with a task complicated by diverse regional specialization that is compounded by intricate cellular complexity (neurons, glia and cell projections) and further exacerbated by synaptic heterogeneity and a huge dynamic range of protein expression. The human brain is composed of an estimated $\sim 10^{12}$ heterogeneous neurons that communicate by way of $\sim 10^{15}$ synapses (Pocklington et al. 2006). Whole brain tissues are variably composed of neurons and glial cells, the latter comprising up to 90-95\% of the cells (Williams et al. 1988). Because most of the glia are astrocytes (Hansson et al. 2003), protein expression analyzed in a whole brain sample may tell us little about neuronal function, in the classic sense. Numerous efforts have been made to establish reference proteomes for brain tissue from various species by surveying the whole brain or gross brain areas (Edgar et al. 1999; Fountoulakis et al. 1999; Gauss et al. 1999; Langen et al. 1999; Beranova-Giorgianni et al. 2002). Our laboratory demonstrated that the expressed proteome can vary in various brain 
regions based on genetic selection for alcohol preference, and, within these genetic lines, by functional nuclei (Witzmann et al. 2003). Despite these documented differences in whole brain tissue, it is likely that many of the proteins previously identified by us and by others in whole brain tissue preparations are of glial, not neuronal origin. For proteomics, a meaningful analysis obligates one to by-pass the whole brain, brain region, and even the micropunch (Leng et al. 2004) or laser-capture (Mouledous et al. 2003; Nazarian et al. 2005) sample. Instead, one must opt for the business-end of the CNS, the synapse.

Synapses are electrical or chemical communicative contacts between neurons. Electrical synapses (neuronal gap junctions) function by the propagation of electrical impulses from one cell to another (and vice versa) via direct, physical contact. As a consequence, these synapses are characterized by a relatively simple organization of membrane structure and associated organelles (Zoidl et al. 2002). Electrical synapses are also less mutable, in terms of their function and molecular characteristics, and thus exhibit little of the plasticity that typifies the chemical synapse.

Characteristically, chemical synapses contain a broad range of chemical neurotransmitters and neuropeptides for intercellular communication, in addition to localized translational machinery that is tightly coupled to signaling (Steward et al. 2003). The latter components make these neuronal junctions particularly relevant to proteomic analysis. Cell-cell communication that occurs by chemical transmission is characterized by complex protein-driven molecular mechanisms of synthesis, delivery, storage, docking, fusion, neurotransmitter release, reuptake, etc. (Purves 2004). In general, synapses are composed of three main constituents: a presynaptic component (presynaptic ending, axon terminal), a synaptic cleft, and a postsynaptic component (dendritic spine). The pre- and the postsynaptic membranes are uniquely distinguishable by visible densities along their corresponding plasma membranes. Together with the synaptic cleft, they are collectively referred to as the synapse (see Figure 1).

Typically, the presynaptic ending is further distinguished from the postsynaptic component by the conspicuous presence of neurotransmitter-filled vesicles. In response to presynaptic membrane depolarization, the vesicles exocytose their contents into the cleft through complicated membrane-trafficking events. The presynaptic axon terminal (bouton) of the presynaptic component also contains other organelles such as mitochondria, smooth endoplasmic reticulum, microtubules, and neurofilaments. The presynaptic membrane is variably populated by docking/fusion apparatus, ion channels, and other protein constituents. The 20-30 nM wide synaptic cleft separates the pre- and postsynaptic membranes and generally contains a dense plaque of intercellular material that includes microfilaments.

The postsynaptic membrane, particularly at the dendritic spine, is recognizable by a collection of dense material visible by electron microscopy on its cytoplasmic surface. This so-called post-synaptic density (PSD) (Palay 1958; Gray 1959) is a specialization of the nerve cell's submembrane cytoskeleton composed of granular/filamentous material, contains cisternae of smooth endoplasmic reticulum, and its existence seems to be dependent on the presence of the presynaptic ending. A subcellular fraction enriched in structures with PSD-like morphology has been shown to contain signal-transduction molecules thought to regulate receptor localization and function in the CNS (Kennedy 1993).

The morphological characteristics of synapses mentioned above reflect the complex functional dynamics of neurotransmission and its plasticity. Though well-studied for many decades, the synaptic molecular anatomy and clues to its complex function in learning/memory, injury, and disease, are just now being unraveled through the application of emerging protein analytical techniques. Four compelling reasons have been identified that underscore the importance of understanding the protein molecular machinery of the synapse (Pocklington et al. 2006): 
1. It is the most important structure for communication between nerve cells.

2. The neurotransmitter receptors and signal transduction machinery within the synapse respond to patterns of electrical activity and instigate biochemical changes in the nerve cell and in so doing modify the brain in response to behavioral experience.

3. Synapse proteins correspond to many human disease genes and drug targets for therapeutics that modulate cognitive illnesses.

4. Synapse proteomic studies have compiled a first draft of the protein composition of the synapse, revealing an unexpectedly high degree of molecular complexity.

The fundamental role of the synapse in neurotransmission and plasticity has fueled increasing proteomic efforts for the identification synaptic protein constituents, leading to the establishment of increasingly comprehensive mapping and profiling of the synaptic proteome. This chapter will summarize a selection of recent studies that have drawn upon established and emerging proteomic technologies, along with fractionation techniques that are essential to the isolation and analysis of specific synaptic components, in an effort to understand the complexity and plasticity of the synapse proteome.

\section{Synaptosome Fractionation}

Sample complexity reduction strategies are required to enable neuroscientists to address the issues mentioned above, and facilitate meaningful applications of proteomic analyses. The feasibility of protein enrichment by subcellular fractionation has been demonstrated through the analysis of the rat brain sub-proteomes of cytosolic, mitochondrial and microsomal fractions (Krapfenbauer et al. 2003). Another well-established subcellular fractionation technique, synaptosomal isolation, has recently been applied in various proteomic studies, providing an enrichment of cellular components found at the synapse. By further fractionating the synaptosomes, one can use proteomic approaches to study even more specific synaptic regions.

\subsection{Synaptosomes}

The term "synaptosome" was first mentioned in a paper published in 1964 by Whittaker's group (Whittaker et al. 1964). At that time, the aim was to explore the synaptosomal localization of several known and putative neurotransmitters and their synthesizing enzymes, and to subfractionate the disrupted synaptosomes to obtain homogeneous fractions of synaptic vesicles and other synaptic components. In this context, rather than organelles, synaptosomes are artificial, membranous sacs that contain synaptic components and are generated by subcellular fractionation of homogenized or ground-up nerve tissue. They are often referred to as "pinched-off nerve endings," because the lipid bilayers naturally reseal together after the axon terminals are torn off by the physical shearing force of homogenization. Synaptosomes contain the complete presynaptic terminal, including mitochondria and synaptic vesicles, along with the postsynaptic membrane and the postsynaptic density (PSD). This typical morphology can be observed clearly via electron microscopy (Figure 2, by permission from (Schrimpf et al. 2005)). Because all the molecular machinery for the release, uptake and storage of neurotransmitters remain intact, synaptosomes are often used to study synaptic transmission.

\subsection{Synaptosome Preparation}

In a manner similar to the isolation of other subcellular fractions containing relatively pure cellular organelles, differential centrifugation or sucrose (Gray and Whittaker 1962; Whittaker et al. 1964), Ficoll/sucrose (Booth et al. 1978), or Percoll (Nagy et al. 1984) density-gradient centrifugation techniques are used for the preparation of synaptosomes. Though the reagents for buffering and for the formation of the density gradient may differ from lab to lab, depending 
on personal preferences or specific applications, all follow the steps involved in purifying a crude synaptosomal fraction from their mitochondrial and microsomal contamination. For instance, here we describe briefly a protocol used in our laboratory for the routine preparation of synaptosomes from fresh brain tissue. Whole brain or a grossly dissected brain region is homogenized in buffer (0.32 M sucrose, $20 \mathrm{mM}$ HEPES, $\mathrm{pH} 7.4$, with proper reducing reagent and protease inhibitors) and centrifuged at $1000 \times \mathrm{g}$ for $10 \mathrm{~min}$ to pellet the membrane fragments and nuclei. The supernatant is then further centrifuged at $17,000 \times \mathrm{g}$ for $15 \mathrm{~min}$ to obtain the pellet containing synaptosomes contaminated with mitochondria and microsomes. This crude synaptosome fraction is then further purified using a discontinuous sucrose density gradient consisting of a $0.8 \mathrm{M}$ sucrose layer on the top and a $1.2 \mathrm{M}$ sucrose layer on the bottom. The crude synaptosome suspension is layered on top of the $0.8 \mathrm{M}$ sucrose layer and centrifuged at $54,000 \times \mathrm{g}$ for $90 \mathrm{~min}$. The pure synaptosomal fraction can be obtained from the interface of $0.8 \mathrm{M}$ sucrose and $1.2 \mathrm{M}$ sucrose. The purity of the isolated synaptosomes can be inspected morphologically under an electron microscope (Figure 2) or analyzed biochemically using enzymatic markers.

\section{The Synaptosomal Proteome}

Comprehensive studies on the synaptic proteome have been rare. Not until quite recently has the mass spectrometric technical momentum developed for detecting and documenting a comprehensive and coherent map of synaptic proteins, currently numbering approximately 1000 unique proteins (Grant 2006). This momentum has been driven by an urgent need in the neuroscientific community for molecular markers of neuropsychiatric and neurodegenerative disorders, as well as a desire to understand the molecular mechanisms underlying synaptic neurotransmission and plasticity. Table 1 presents a compact list of recent proteomic efforts where protein fractions were derived from synapses, and the proteomic methods used to study them. These studies are described further in the text that follows.

\subsection{Sample Complexity Reduction and Protein Enrichment}

In addition to the direct analysis of the synaptosomal proteome (Boyd-Kimball et al. 2005; Schrimpf et al. 2005; Witzmann et al. 2005), protein subsets have been extracted and examined either as a subcompartment or subfraction of synaptosomes, such as synaptic vesicles (Coughenour et al. 2004; Morciano et al. 2005), or from a particular structural component, such as synaptic membranes (Stevens et al. 2003), presynaptic particles (Phillips et al. 2005), synaptodendrosomes (dendritic spine preparations) (Rao et al. 1993; Leski et al. 1996; Villanueva et al. 2001; Jiang et al. 2002), and PSD (Walikonis et al. 2000; Satoh et al. 2002; Li et al. 2004; Peng et al. 2004; Yoshimura et al. 2004; Phillips et al. 2005; Cheng et al. 2006; Dosemeci et al. 2006; Jordan et al. 2006). The analysis of these subproteomes holds great promise for improving our understanding of the temporal and spatial processes that coordinate synaptic proteins in closely related complexes under both normal physiological or pathological conditions.

Synaptosomal proteins also can be enriched by immunoprecipitation and/or affinity purification. Immunoprecipitation uses highly specific antibodies to remove individual target proteins or groups of related proteins (with similar antigenicity) from a complex mixture. Often, those proteins closely interacting with the target protein(s) are also recovered, making this a commonly used method to study protein-protein interactions (Phizicky, et al. 1995). Affinity purification purifies the protein of interest, such as antibodies, fusion-tagged proteins, and biotinylated proteins by virtue of its specific binding properties to an immobilized ligand. For example, Concanavalin A (Con A) is a tetrameric metalloprotein that binds sugar moieties with C-3, C-4 and C-5 hydroxyl groups. Con A coupled to Sepharose is routinely used for the separation and purification of glycoproteins, specifically for proteomic analysis. The power of sample complexity reduction and protein enrichment has been demonstrated by the analysis 
of PSD components using the combination of subcellular fractionation, immunoaffinity purification and LC-MS/MS, where more than 400 PSD components (Walikonis et al. 2000; Jordan et al. 2004; Li et al. 2004; Peng et al. 2004; Yoshimura et al. 2004; Jordan et al. 2006) and 186 NMDA receptor-associated proteins (Husi et al. 2000) have been isolated and identified.

Another subsynaptic fraction derived directly from presynaptic specializations, the presynaptic particle fraction (PPF) can be separated from PSD by adjusting the $\mathrm{pH}$ of Triton X-100 extraction of isolated trans-synaptic scaffolds. As one might expect, it has been shown that the major proteins of the PPF, clathrin and dynamin, are concentrated in the presynaptic compartment (Phillips et al. 2005). These investigators used multidimensional protein identification technology (MudPIT) to compare the PPF and the PSD fraction. Of 341 proteins identified, 50 localized in the PPF, 231 in the PSD fraction, and 60 were found to be common to both fractions. The PPF was also characterized by a low proportion of actin and actinassociated proteins along with a high proportion of vesicle proteins. The authors concluded that the PPF consists of presynaptic proteins not connected to the actin-based synaptic framework and that clathrin may be an anchorage scaffold for many presynaptic proteins.

\subsection{Proteomic Analysis Platforms}

The various technical approaches used in proteomics for synaptosomal proteome analysis are addressed throughout this volume and therefore will not be formally described or listed here. Whether the approach is gel-based, mass spec-based, uses stable-isotopic labels, or is labelfree; each has its own unique merits and deficiencies, and many of the latter are shared among them all. Consequently, the suitability of a chosen technique can dramatically affect the outcome of a study and experimental design must therefore be done with great care. The combination of specific protein enrichment/complexity reduction strategies with appropriate protein resolution (one- and two-dimensional gel electrophoresis (2-DE), solution isoelectric focusing (sIEF), liquid chromatography (LC)) and identification (peptide mass fingerprinting (PMF), tandem mass spectrometry (MS/MS), Western blotting) techniques for that protein subset is the key to achieving an optimal result. Sometimes, the simplest approach (e.g. Western blotting) can be highly informative (see Figure 3 below; (Jordan et al. 2004)). Similar enrichment of PSD proteins has been demonstrated in this manner (Morciano et al. 2005; Cheng et al. 2006; Trinidad et al. 2006).

When a global assessment of the total synaptosome proteome is desired, a combination of several proteomic platforms should be used, as these have been shown to be complementary rather than alternative ways of measuring protein abundances in biological systems (Wu et al. 2006). The commonly used protein identification techniques include matrix-assisted laserdesorption ionization (MALDI) based PMF and LC-MS/MS sequencing. LC-MS/MS sequencing is particularly useful for direct protein identification or for robust confirmation of protein IDs derived from PMF. 2-DE-based protein separation followed by the MS identification of differentially expressed proteins remains the most commonly used platform for the quantitation of differentially expressed proteins, particularly for a multi-group comparison. Other quantitative proteomic approaches, such as 2-D (Fluorescence) Difference Gel Electrophoresis (2D-DIGE, see below), the Isotope-Coded Affinity Tag (ICAT) reagent approach, and label free-quantitative proteomics are in continued development and improvement, aiming to address the problems associated with the traditional 2-DE gel based assays.

\section{Technical Challenges of Synaptosomal Proteomics}

A significant challenge for neuroscientists in studying the membranous synaptosomal proteome rests squarely in the analysis of its constituent hydrophobic and membrane-bound 
proteins. These include receptors, transporters, ion channels, and the molecular machinery for synaptic vesicle cycling. Hydrophobic and membrane-bound proteins are poorly resolved by traditional IEF gel technology. Similarly, they tend to resist in-gel tryptic digestion, leading to poor rates of protein identification by PMF (van Montfort et al. 2002).

Acidic PAGE systems using cationic detergents such as benzyldimethyl- $n$ hexadecylammonium chloride (16-BAC) are useful for resolving basic and membrane proteins. Using a cationic 16-BAC polyacrylamide gel system in the first and SDS-PAGE in the second dimension, immunoaffinity purified pools of mouse brain synaptic vesicles have been analyzed successfully (Kramer 2006). Many integral and membrane associated proteins were identified from a fraction containing free synaptic vesicles and a second fraction containing synaptic vesicles plus presynaptic membranes, using either MALDI-TOF or Western Blot analysis. Some of the proteins detected included the integral synaptic vesicle proteins such as SV2A and SV2B, the zinc transporter ZnT-3, synaptotagmins I and II, synaptophysin, v-SNARE VAMP II, t-SNARE syntaxin 1B2 and SNAP-25, vesicular neurotransmitter transporters for acetylcholine (vAChT), glutamate (vGluT1), and GABA (vGAT), the proton pumping vacuolar ATPase (V1: A1, B1, B2, C1, D, E1, E2, G2; V0: a, d1), peripheral synaptic vesicle proteins such as the synapsins Ia, Ib, IIa and IIb, alpha subunit of calcium/calmodulin dependent kinase II, proteins transiently associated with the synaptic vesicle compartment such as syntaxin binding protein 1 , N-ethylamide sensitive factor (NSF), munc18, munc13, and presynaptic membrane protein $\mathrm{N}$-type $\mathrm{Ca}^{2+}$-channel.

In addition to the inherent difficulty of resolving membrane and basic proteins using 2-DE, gel-gel reproducibility also has been a significant challenge. 2D-DIGE technology was developed to address the gel variability issue. In 2D-DIGE analysis, two samples for comparison are labeled respectively with one of the CyDye fluors. The mixture of the labeled samples, together with a labeled standard can be resolved on a single 2-D gel (Unlu et al. 1997). Identical protein from different samples labeled with each of the fluors migrates to the same position on a 2-D gel, enabling the analysis of differences in protein abundance by comparing the fluorescent intensity of the overlaying protein spots, and subsequent identification by MS techniques. Unfortunately, this technique is only feasible for the simultaneous comparison of sample pairs. Though the application of 2D-DIGE has not yet been reported in the analysis of synaptosomal proteins, a recent study using this technique for the detection of phosphorylation pattern changes in rat cortical neuron cultures upon phosphatase treatment has been conducted (Raggiaschi et al. 2006), and its potential relevance to synaptosomal studies is clear.

Another technical challenge using the 2-DE-based approach relates to its narrow dynamic range of protein detection, a limitation that makes it almost impossible to detect and compare the expression of many very low abundance proteins, even with sample subfractionation. Though various aforementioned protein enrichment techniques are available, the application of LCbased protein separation coupled with highly sensitive mass spectrometric protein identification can address the issue of dynamic range, to a certain extent. This approach also improves the resolution and identification of proteins with extreme pI, large mass, and, in some cases, membrane associations.

For differential, relative protein quantitation, ICAT reagents have been employed in concert with tandem mass spectrometry, enabling LC-MS/MS based analysis of differentially expressed proteins from different physiological and pathological states (Gygi et al. 1999). Each ICAT reagent contains an affinity tag (biotin moiety), an isotopically labeled linker (contains heavy or light stable isotopes, such as d8-heavy or d0-light), and a chemical reactive group (such as a thiol-specific reactive group). After the free thiol groups of cysteine residues from each of the two populations of proteins are tagged with a heavy or a light ICAT reagent, same 
amount of proteins are combined and tryptic digested, followed by affinity enrichment of the labeled peptides using an avidin column. These peptides are then analyzed by LC-MS/MS. The ratios of signal intensities of differentially mass-tagged peptide pairs are quantified to determine the relative levels of proteins in the two samples. The sensitivity and reliability of the technique have been further improved by the generation of cleavable ICAT reagents (Hansen et al. 2003).

In a recent study, the ICAT reagent was employed for the proteomic characterization of synaptosomes isolated from mouse brain (Schrimpf et al. 2005). Though only cysteine containing proteins were analyzed, more than a thousand proteins were identified by LC-MS/ MS, including proteins involved in synaptic vesicle exocytosis, synaptic vesicle recycling, postsynaptic receptors and proteins constituting the PSD, as well as a large number of soluble and membrane-bound proteins for synaptic signal transduction and metabolism. In a similar application, the effect of chronic morphine exposure on the synaptic plasma-membrane subproteome in rats was studied using ICAT technology (Prokai et al. 2005). These researchers observed a number of significant expression changes including the down-regulation of an integral membrane protein $\mathrm{Na}^{+} / \mathrm{K}^{+}$ATPase ( $\alpha$-subunit) involved in regulation of the cell membrane potential that correlated to a decline in electrogenic $\mathrm{Na}^{+}, \mathrm{K}^{+}$pumping of identical magnitude.

More recently, a study was conducted on the rat brain to compare the regional differences in the protein expression profile of PSD proteins between forebrain and cerebellum (Cheng et al. 2006). LC-MS/MS analysis of ICAT reagent-labeled peptides (including the absolute quantification, AQUA, strategy) revealed crucial molecular differences in PSD between the brain areas. Among 296 proteins identified and quantified, 43 differed significantly in abundance. These included proteins involved in cell adhesion, cytoskeleton/cellular scaffold, GTPase regulation, kinase/phosphatase regulation, membrane trafficking, mitochondria, motor function, receptors and channels, metabolism, and others. The differences in the expression of Nir2 (phosphatidyl inositol transfer protein), Septin 4, and GluR $\delta$ were further confirmed by immunostaining of sagittal sections of rat brain.

It bears repeating that all proteomic approaches have an intrinsic bias toward the detection of a certain subset or type of proteins. No single approach has the capacity for a truly comprehensive analysis of the total proteome. Therefore, it is necessary to combine several technical approaches to even approximate a global analysis. Our laboratory has analyzed the proteome of rat cortical synaptosomes using two types of proteomic approaches. The first, 2DE separation coupled with MALDI-TOF and LC-MS/MS identification of proteins from gel excisions detected a total of 968 protein spots. The 94 most abundant protein spots were analyzed by MALDI-based PMF resulting in the identification of 85, representing 61 unique proteins. Another 96 low-abundance protein spots were analyzed by LC-MS/MS, yielding 79 identifications and representing 46 additional unique proteins. A total of 19 unique proteins thus were identified using the initial approach.

The second approach, a shotgun proteomic analysis (LC-MS/MS) of tryptic solution-digests of isolated synaptosomes, identified 209 unique proteins. When the two sets of identified proteins are compared, 46 were found to be common. Accounting for this intersection, the total number of unique proteins identified by the combined methods was 254 . Of these 254 proteins, 61 were identified by PubMed literature search as having synapse-specific function. A total of 19 identified proteins were involved in synaptic vesicle trafficking or docking, 9 served receptor or transporter functions, 9 were involved in intra-cellular signaling cascades that affect synaptic transmission, and 24 had other synapse-specific functions. Figure 4; (see colour insert) illustrates the major pre- and postsynaptic proteins identified by 2-DE/MS and/or 
shotgun proteomics in this study, a set of proteins normally expected as constituent in synaptosomal preparations (Witzmann et al. 2005).

\section{Delineation of the Functional Relationships of Synaptic Proteins - Post- Translational Modifications}

Many published studies have significantly improved our understanding of synaptic function by describing the protein constituency of synaptosomes, and the number of these documented synaptic proteins is rapidly expanding. However, more efforts are needed for an integrated functional understanding of the synapse. The analysis of the changes in the state of protein post-translational modification (PTM) is an effective approach for this purpose because PTM regulates nearly every aspect of synaptic function, including neurotransmitter release, synaptic vesicle recycling, receptor/ion channel function, and local protein synthesis (Barry et al. 2002; Lisman et al. 2002; Korolchuk et al. 2003; Purcell et al. 2003; Waltereit et al. 2003; Han et al. 2004).

PTM also plays a critical role in synaptic plasticity and memory (Purcell et al. 2003; Routtenberg et al. 2005). For instance, tyrosine kinase signal transduction cascades of Trk receptor tyrosine kinases, the Src family of non-receptor tyrosine kinases and the Eph receptor tyrosine kinases have been shown to associate with the synaptic plasticity and memory processing (Purcell et al. 2003). The main protein constituent of PSD, calcium/calmodulindependent protein kinase II (CaMKII) has been proposed to act as a bi-stable switch by auto phosphorylation/dephosphorylation for the long-term storage of synaptic memory (Lisman et al. 2002). The activity of CaMKII is regulated by $\mathrm{Ca}^{2+} /$ calmodulin, where $\mathrm{Ca}^{2+}$ release is controlled by NMDA receptor activation. CaMKII dictates synaptic strength, mainly by affecting the functional properties of AMPA ( $\alpha$-amino-3-hydroxy-5-methyl-4-isoxazole propionic acid) receptors, as well as their trafficking and anchorage to the postsynaptic membrane (Barry et al. 2002).

Clathrin-mediated endocytosis is a major vesicular transport mechanism in the neuron, which enables the internalization of plasma membrane-bound proteins, nutrients, hormones and other molecules associated with the plasma membrane into intracellular compartments. Clathrin and various adaptor and accessory proteins work in concert at different stages of clathrin coated vesicle formation and disassembly, and many of these proteins (such as clathrin light chain, AP-2, dynamin 1, synaptojanin 1, and the amphiphysins) are substrates for protein kinases (Korolchuk et al. 2003). In addition, it has been suggested that directing synaptotagmin 1 to the synaptic vesicle is dependent on the $\mathrm{N}$-terminal glycosylation of this protein (Han et al. 2004).

Using immobilized metal affinity chromatography (IMAC), phosphopeptides haven been isolated and enriched from tryptically digested proteins extracted from freshly isolated human cortical synaptosomes (DeGiorgis et al. 2005). LC-MS/MS analysis identified 26 synaptic proteins with 1, 2 or 3 phosphoserine(s), including synapsin 1, syntaxin 1, SNIP, PSD-93, NCAM, GABA-B receptor, chaperone molecules, and protein kinases. In another study (Collins et al. 2005a), LC-MS/MS analysis of IMAC enriched phosphopeptides from mouse forebrain synaptosomes, identified 228 potential synaptic phosphoproteins and characterized over 350 phosphopeptides containing 331 sites of phosphorylation, including the identification of highly phosphorylated MAP1B (33 sites) and Bassoon (30 sites). Bioinformatic tools, such as Scansite (www.scansite.mit.edu/) and Net-PhosK (www.cbs.dtu.dk/services/NetPhos/), were employed to predict the likeliness of these identified proteins to be phosphorylated, the possible phosphorylation sites and the responsible kinases. Additionally, protein-protein interaction database (www.ppid.org) was utilized to analyze the protein-protein interaction of the NMDA receptor. 
Fractionation of proteins by strong cation exchange (SCX) chromatography, followed by IMAC enrichment of phosphopeptides from SCX fractions, led to a comprehensive identification of phosphoproteins of PSD isolated from mouse brain using LC-MS/MS (Trinidad et al. 2006). In this study, phosphorylation site(s) were mapped to 287 proteins from a total of 1,264 unique proteins identified. This translates into a 23\% phosphorylation rate, comparable to an expected 33\% rate in the general proteome (Johnson et al. 2005). The 287 phosphoproteins were derived from a total of 998 unique phosphorylated peptides, and the phosphorylations were mapped to 723 unique sites. Most of these occurred on serines, to a lesser extent on threonines, and only minimally on tyrosines (Figure 5A; see colour insert).

Proteins within a broad range of functional categories were involved, including structural proteins, scaffolding proteins, kinases and phosphatases, membrane neurotransmitter receptors, and voltage-gated ion channels (Figure 5B). When the number of unique phosphorylation sites, was analyzed, as a function of the protein functional class, adhesion/ cytoskeleton proteins appeared to carry the most modification followed by adaptor/sorting proteins, presynaptic proteins, and kinases/phosphatases (Figure 5B).

Another PMT with functional importance and complex interplay with phosphorylation in the brain is the cytosolic- and nuclear-specific $O$-linked $N$-acetylglucosamine ( $O$-GlcNAc) glycosylation. This is an abundant, dynamic, and inducible carbohydrate modification of serines and threonines by $\mathrm{N}$-acetylglucosamine that plays a role in protein regulation of cellular signaling networks, nuclear transcription, protein turnover, and the cell cycle (Slawson et al. 2006). $O$-GlcNAc transferase (OGT) and $O$-GlcNAcase, which are responsible for the addition and removal of $O$-GlcNac protein modifications, are highly expressed in the brain (Gao et al. 2001; Iyer et al. 2003).

Recently, a chemoenzymatic approach was employed for the identification of $O$-GlcNAc modified proteins from rat brain. This approach allows the transfer of a ketone-containing galactose analogue selectively to the C-4 hydroxyl of GlcNAc by an engineered $\alpha-1,4-$ galactosyltransferase enzyme. The ketone functionality is further reacted with an aminooxy biotin nucleophile, which enables the enrichment of $O$-GlcNAc modified peptides using avidin affinity chromatography. Using this approach, 25 unique proteins were identified from rat forebrain, including 23 newly identified and 2 previously reported proteins in $O$-GlcNAcglycosylated form (microtubule-associated protein 2B and host cell factor) (Khidekel et al. 2004). As expected, these proteins were functionally categorized as proteins associated with gene expression, neuronal signaling, and synaptic plasticity.

Using in situ hybridization and immuno-labeling, Akimoto et al. (2003) found OGT transcripts and proteins to be enriched in the cortical neurons of rat cerebellum, particularly in the Purkinje cells. In nerve terminals, the OGT enzyme was observed around synaptic vesicles, in the preand post-synaptic terminals. The localization of immuno-labeled $O$-GlcNAc was also in general agreement with the distribution of OGT. In a related study, abundant OGT and $O$ GlcNAcase activities were detected in intact, functional synaptosomes, particularly in the soluble portion of the synaptosomal fraction (Cole et al. 2001). Using wheat germ agglutininbased lectin weak affinity chromatography for the enrichment of $O$-GlcNAc modified peptides, 145 unique $O$-GlcNAc modified peptides were identified from a PSD preparation of mouse brain (Vosseller et al. 2006). Sixty five of these $O$-GlcNAc modified peptides were sequenced, and belonged to such functional classes as synapse assembly, synaptic vesicle trafficking, and post-synaptic signaling.

It is widely acknowledged that the efficient implementation of currently available and newly emerging bioinformatic tools has become critical for the extraction of physiological and biological principles from extremely complex proteomic data sets. This, in turn, can facilitate 
the elucidation of mechanisms and disease processes, and the development of effective therapeutics. Continuous improvement in proteomic technologies has facilitated a rapid expansion of identified synaptic components. However, the biological interpretation of these data has lagged behind. Systems Biology approaches (Ideker et al. 2001; Grant 2003; Jordan et al. 2006) that seek to integrate multiple levels of information (e.g. gene and protein networks) to understand how biological systems function, are needed to overcome this deficiency. Exactly such an approach has been recently applied for the analysis of NMDA receptor complex, using the protein-protein interaction network maps and annotated functions of individual components (Pocklington et al. 2006).

\section{Conclusion}

A significant goal of understanding synaptic molecular composition and function is to determine the pathogenic mechanisms through which neuropsychological disorders develop, and eventually to devise efficacious interventions, be they biological or pharmaceutical. To achieve this goal, the mapping and profiling of structural and functional synaptic protein components is merely a first step. Reliable and reproducible determination of quantitative or qualitative changes, or both, in these components is ultimately essential. With recent technological advances that have produced mass spectrometers with unprecedented sensitivity/ resolution, LC apparatus with great flow stability and compositional accuracy, and autosamplers with very high injection precision, our knowledge of identified synaptic components and their myriad modifications has exploded. This chapter has merely touched the surface of synaptic molecular complexity. In doing so, we hope it has illuminated the tremendous possibilities that lie ahead for neuroscientists when the powerful tools of cellular subfractionation and proteomics are skillfully combined.

\section{References}

Akimoto Y, Comer FI, et al. Localization of the O-GlcNAc transferase and O-GlcNAc-modified proteins in rat cerebellar cortex. Brain Res 2003;966:194-205. [PubMed: 12618343]

Barry MF, Ziff EB. Receptor trafficking and the plasticity of excitatory synapses. Curr Opin Neurobiol 2002;12:279-286. [PubMed: 12049934]

Beranova-Giorgianni S, Pabst MJ, et al. Preliminary analysis of the mouse cerebellum proteome. Brain Res Mol Brain Res 2002;98:135-140. [PubMed: 11834305]

Booth RF, Clark JB. A rapid method for the preparation of relatively pure metabolically competent synaptosomes from rat brain. Biochem J 1978;176:365-370. [PubMed: 743245]

Boyd-Kimball D, Castegna A, et al. Proteomic identification of proteins oxidized by A[beta] (1-42) in synaptosomes implications for Alzheimer's disease. Brain Res 2005;1044:206-215. [PubMed: 15885219]

Cheng D, Hoogenraad CC, et al. Relative and absolute quantification of postsynaptic density proteome isolated from rat forebrain and cerebellum. Mol Cell Proteomics 2006;5:1158-1170. [PubMed: 16507876]

Cole RN, Hart GW. Cytosolic O-glycosylation is abundant in nerve terminals. J Neurochem 2001;79:1080-1089. [PubMed: 11739622]

Collins MO, Yu L, et al. Proteomic analysis of in vivo phosphorylated synaptic proteins. J Biol Chem 2005a;280:5972-5982. [PubMed: 15572359]

Collins MO, Yu L, et al. Robust enrichment of phosphorylated species in complex mixtures by sequential protein and peptide metal-affinity chromatography and analysis by tandem mass spectrometry. Sci STKE 2005b;2005:16.

Coughenour HD, Spaulding RS, et al. The synaptic vesicle proteome: a comparative study in membrane protein identification. Proteomics 2004;4:3141-3155. [PubMed: 15378707]

DeGiorgis JA, Jaffe H, et al. Phosphoproteomic Analysis of Synaptosomes from Human Cerebral Cortex. J Proteome Res 2005;4:306-315. [PubMed: 15822905] 
Dosemeci A, Tao-Cheng JH, et al. Preparation of postsynaptic density fraction from hippo-campal slices and proteomic analysis. Biochem Biophys Res Commun 2006;339:687-694. [PubMed: 16332460]

Edgar PF, Douglas JE, et al. Proteome map of the human hippocampus. Hippocampus 1999;9:644-650. [PubMed: 10641757]

Fountoulakis M, Schuller E, et al. Rat brain proteins: two-dimensional protein database and variations in the expression level. Electrophoresis 1999;20:3572-3579. [PubMed: 10612283]

Gao Y, Wells L, et al. Dynamic O-glycosylation of nuclear and cytosolic proteins: cloning and characterization of a neutral, cytosolic beta- $\mathrm{N}$-acetylglucosaminidase from human brain. J Biol Chem 2001;276:9838-9845. [PubMed: 11148210]

Gauss C, Kalkum M, et al. Analysis of the mouse proteome (I) Brain proteins: separation by twodimensional electrophoresis and identification by mass spectrometry and genetic variation. Electrophoresis 1999;20:575-600. [PubMed: 10217174]

Grant SG. Systems biology in neuroscience: bridging genes to cognition. Curr Opin Neurobiol 2003;13:577-582. [PubMed: 14630221]

Grant SG. The synapse proteome and phosphoproteome: a new paradigm for synapse biology. Biochem Soc Trans 2006;34:59-63. [PubMed: 16417483]

Gray EG. Axo-somatic and axo-dendritic synapses of the cerebral cortex: an electron microscope study. J Anat 1959;93:420-433. [PubMed: 13829103]

Gray EG, Whittaker VP. The isolation of nerve endings from brain: an electron-microscopic study of cell fragments derived by homogenization and centrifugation. J Anat 1962;96:79-88. [PubMed: 13901297]

Gygi SP, Rist B, et al. Quantitative analysis of complex protein mixtures using isotope-coded affinity tags. Nat Biotechnol 1999;17:994-999. [PubMed: 10504701]

Han W, Rhee JS, et al. $N$-glycosylation is essential for vesicular targeting of synaptotagmin 1 . Neuron 2004;41:85-99. [PubMed: 14715137]

Hansen KC, Schmitt-Ulms G, et al. Mass spectrometric analysis of protein mixtures at low levels using cleavable 13C-isotope-coded affinity tag and multidimensional chromatography. Mol Cell Proteomics 2003;2:299-314. [PubMed: 12766231]

Hansson E, Ronnback L. Glial neuronal signaling in the central nervous system. FASEB J 2003;17:341348. [PubMed: 12631574]

Husi H, Ward MA, et al. Proteomic analysis of NMDA receptor-adhesion protein signaling complexes. Nat Neurosci 2000;3:661-669. [PubMed: 10862698]

Ideker T, Galitski T, et al. A new approach to decoding life: systems biology. Annu Rev Genomics Hum Genet 2001;2:343-372. [PubMed: 11701654]

Iyer SP, Hart GW. Dynamic nuclear and cytoplasmic glycosylation: enzymes of O-GlcNAc cycling. Biochemistry 2003;42:2493-2499. [PubMed: 12614143]

Jiang C, Schuman EM. Regulation and function of local protein synthesis in neuronal dendrites. Trends in Biochem Sci 2002;27:506-513. [PubMed: 12368086]

Johnson SA, Hunter T. Kinomics: methods for deciphering the kinome. Nat Methods 2005;2:17-25. [PubMed: 15789031]

Jordan B, Ziff E. Getting to synaptic complexes through systems biology. Genome Biol 2006;7:214. [PubMed: 16677427]

Jordan BA, Fernholz BD, et al. Identification and Verification of Novel Rodent Postsynaptic Density Proteins. Mol Cell Proteomics 2004;3:857-871. [PubMed: 15169875]

Jordan, BA.; Fernholz, BD., et al. New tricks for an old dog: proteomics of the PSD. In: Kittler, JT.; Moss, SJ., editors. The Dynamic Synapse: Molecular Methods in Ionotropic Receptor Biology. Boca Raton; CRC/Taylor \& Francis: 2006. p. 37-55.

Kennedy MB. The postsynaptic density. Curr Opin Neurobiol 1993;3:732-737. [PubMed: 8260822]

Khidekel N, Ficarro SB, et al. Exploring the O-GlcNAc proteome: direct identification of O-GlcNAcmodified proteins from the brain. Proc Natl Acad Sci USA 2004;101:13132-13137. [PubMed: 15340146]

Kim SI, Voshol H, et al. Neuroproteomics: expression profiling of the brain's proteomes in health and disease. Neurochem Res 2004;29:1317-1331. [PubMed: 15176488] 
Korolchuk V, Banting G. Kinases in clathrin-mediated endocytosis. Biochem Soc Trans 2003;31:857860. [PubMed: 12887321]

Kramer ML. A new multiphasic buffer system for benzyldimethyl- $n$-hexadecylammonium chloride polyacrylamide gel electrophoresis of proteins providing efficient stacking. Electrophoresis 2006;27:347-356. [PubMed: 16331586]

Krapfenbauer K, Fountoulakis M, et al. A rat brain protein expression map including cytosolic and enriched mitochondrial and microsomal fractions. Electrophoresis 2003;24:1847-1870. [PubMed: 12783461]

Langen H, Berndt P, et al. Two-dimensional map of human brain proteins. Electrophoresis 1999;20:907916. [PubMed: 10344266]

Leng A, Feldon J, et al. Long-term social isolation and medial prefrontal cortex: dopaminergic and cholinergic neurotransmission. Pharmacol Biochem Behav 2004;77:371-379. [PubMed: 14751467]

Leski ML, Steward O. Protein synthesis within dendrites: ionic and neurotransmitter modulation of synthesis of particular polypeptides characterized by gel electrophoresis. Neurochem Res 1996;21:681-690. [PubMed: 8829140]

Li KW, Hornshaw MP, et al. Proteomics analysis of rat brain postsynaptic density. Implications of the diverse protein functional groups for the integration of synaptic physiology. J Biol Chem 2004;279:987-1002. [PubMed: 14532281]

Lisman J, Schulman H, et al. The molecular basis of CaMKII function in synaptic and behavioural memory. Nat Rev Neurosci 2002;3:175-190. [PubMed: 11994750]

Morciano M, Burre J, et al. Immunoisolation of two synaptic vesicle pools from synaptosomes: a proteomics analysis. J Neurochem 2005;95:1732-1745. [PubMed: 16269012]

Mouledous L, Hunt S, et al. Proteomic analysis of immunostained, laser-capture microdissected brain samples. Electrophoresis 2003;24:296-302. [PubMed: 12652601]

Nagy A, Delgado-Escueta AV. Rapid preparation of synaptosomes from mammalian brain using nontoxic isoosmotic gradient material (Percoll). J Neurochem 1984;43:1114-1123. [PubMed: 6088694]

Nazarian J, Bouri K, et al. Intracellular expression profiling by laser capture microdissection: three novel components of the neuromuscular junction. Physiol Genomics 2005;21:70-80. [PubMed: 15623565]

Palay SL. The morphology of synapses in the central nervous system. Exp Cell Res 1958;14:275-293. [PubMed: 13586295]

Peng J, Kim MJ, et al. Semiquantitative proteomic analysis of rat forebrain postsynaptic density fractions by mass spectrometry. J Biol Chem 2004;279:21003-21011. [PubMed: 15020595]

Phillips GR, Florens L, et al. Proteomic comparison of two fractions derived from the transsynaptic scaffold. J Neurosci Res 2005;81:762-775. [PubMed: 16047384]

Phizicky EM, Fields S. Protein-protein interactions: methods for detection and analysis. Microbiol Rev 1995;59:94-123. [PubMed: 7708014]

Pocklington AJ, Armstrong JD, et al. Organization of brain complexity—synapse proteome form and function. Brief Funct Genomic Proteomic 2006;5:66-73. [PubMed: 16769682]

Prokai L, Zharikova AD, et al. Effect of chronic morphine exposure on the synaptic plasma-membrane subproteome of rats: a quantitative protein profiling study based on isotope-coded affinity tags and liquid chromatography/mass spectrometry. J Mass Spectrom 2005;40:169-175. [PubMed: 15706614]

Purcell AL, Carew TJ. Tyrosine kinases, synaptic plasticity and memory: insights from vertebrates and invertebrates. Trends Neurosci 2003;26:625-630. [PubMed: 14585603]

Purves, D. Neuroscience. Sunderland, Massachusetts: Sinauer Associates; 2004.

Raggiaschi R, Lorenzetto C, et al. Detection of phosphorylation patterns in rat cortical neurons by combining phosphatase treatment and DIGE technology. Proteomics 2006;6:748-756. [PubMed: 16372257]

Rao A, Steward O. Evaluation of RNAs present in synaptodendrosomes: dendritic, glial, and neuronal cell body contribution. J Neurochem 1993;61:835-844. [PubMed: 7689643]

Routtenberg A, Rekart JL. Post-translational protein modification as the substrate for long-lasting memory. Trends Neurosci 2005;28:12-19. [PubMed: 15626492] 
Satoh K, Takeuchi M, et al. Identification of activity-regulated proteins in the postsynaptic density fraction. Genes Cells 2002;7:187-197. [PubMed: 11895482]

Schrimpf SP, Meskenaite V, et al. Proteomic analysis of synaptosomes using isotope-coded affinity tags and mass spectrometry. Proteomics 2005;5:2531-2541. [PubMed: 15984043]

Slawson C, Housley MP, et al. O-GlcNAc cycling: how a single sugar post-translational modification is changing the way we think about signaling networks. J Cell Biochem 2006;97:71-83. [PubMed: 16237703]

Stevens SM Jr, Zharikova AD, et al. Proteomic analysis of the synaptic plasma membrane fraction isolated from rat forebrain. Brain Res Mol Brain Res 2003;117:116-128. [PubMed: 14559145]

Steward O, Schuman EM. Compartmentalized synthesis and degradation of proteins in neurons. Neuron 2003;40:347-359. [PubMed: 14556713]

Trinidad JC, Specht CG, et al. Comprehensive Identification of Phosphorylation Sites in Postsynaptic Density Preparations. Mol Cell Proteomics 2006;5:914-922. [PubMed: 16452087]

Unlu M, Morgan ME, et al. Difference gel electrophoresis: a single gel method for detecting changes in protein extracts. Electrophoresis 1997;18:2071-2077. [PubMed: 9420172]

van Montfort BA, Canas B, et al. Improved in-gel approaches to generate peptide maps of integral membrane proteins with matrix-assisted laser desorption/ionization time-of-flight mass spectrometry. J Mass Spectrom 2002;37:322-330. [PubMed: 11921374]

Villanueva S, Steward O. Protein synthesis at the synapse: developmental changes, subcellular localization and regional distribution of polypeptides synthesized in isolated dendritic fragments. Brain Res Mol Brain Res 2001;91:148-153. [PubMed: 11457502]

Vosseller K, Trinidad JC, et al. O-Linked N-Acetylglucosamine proteomics of postsynaptic density preparations using lectin weak affinity chromatography and mass spectrometry. Mol Cell Proteomics 2006;5:923-934. [PubMed: 16452088]

Walikonis RS, Jensen ON, et al. Identification of proteins in the postsynaptic density fraction by mass spectrometry. J Neurosci 2000;20:4069-4080. [PubMed: 10818142]

Waltereit R, Weller M. Signaling from cAMP/PKA to MAPK and synaptic plasticity. Mol Neurobiol 2003;27:99-106. [PubMed: 12668903]

Whittaker VP, Michaelson IA, et al. The separation of synaptic vesicles from nerve-ending particles (“synaptosomes"). Biochem J 1964;90:293-303. [PubMed: 5834239]

Williams RW, Herrup K. The control of neuron number. Annu Rev Neurosci 1988;11:423-453. [PubMed: 3284447]

Witzmann FA, Arnold RJ, et al. A proteomic survey of rat cerebral cortical synaptosomes. Proteomics 2005;5:2177-2201. [PubMed: 15852343]

Witzmann FA, Li J, et al. Innate differences in protein expression in the nucleus accumbens and hippocampus of inbred alcohol-preferring and -nonpreferring rats. Proteomics 2003;3:1335-1344. [PubMed: 12872235]

Wu WW, Wang G, et al. Comparative study of three proteomic quantitative methods, DIGE, cICAT, and iTRAQ, using 2D gel- or LC-MALDI TOF/TOF. J Proteome Res 2006;5:651-658. [PubMed: 16512681]

Yoshimura Y, Yamauchi Y, et al. Molecular constituents of the postsynaptic density fraction revealed by proteomic analysis using multidimensional liquid chromatography-tandem mass spectrometry. $\mathrm{J}$ Neurochem 2004;88:759-768. [PubMed: 14720225]

Zoidl G, Dermietzel R. On the search for the electrical synapse: a glimpse at the future. Cell Tissue Res 2002;310:137-142. [PubMed: 12397368] 


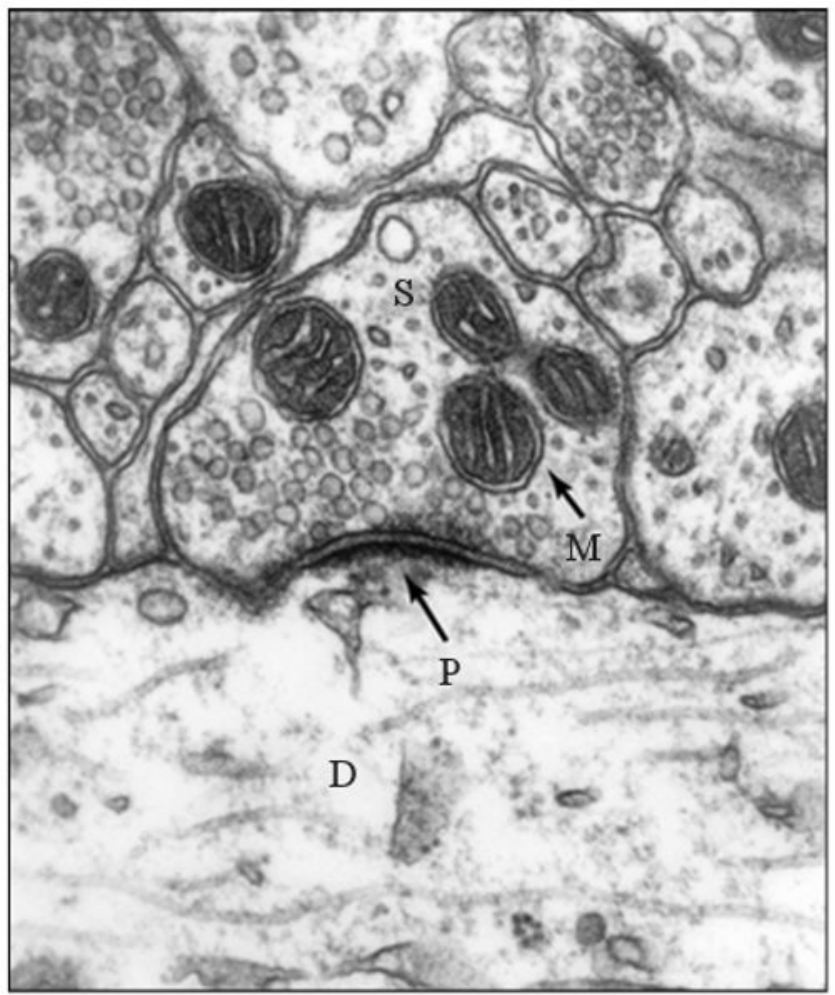

Figure 1.

Electron photomicrograph a synapse $(56,000 \times)$ illustrating the synaptic knob $(\mathrm{S})$ as it ends on the shaft of a dendrite (D) in the central nervous system. P, postsynaptic density; M, mitochondrion. (From: Review of Medical Physiology, 22nd Edition, The McGraw-Hill Co., 2005) 


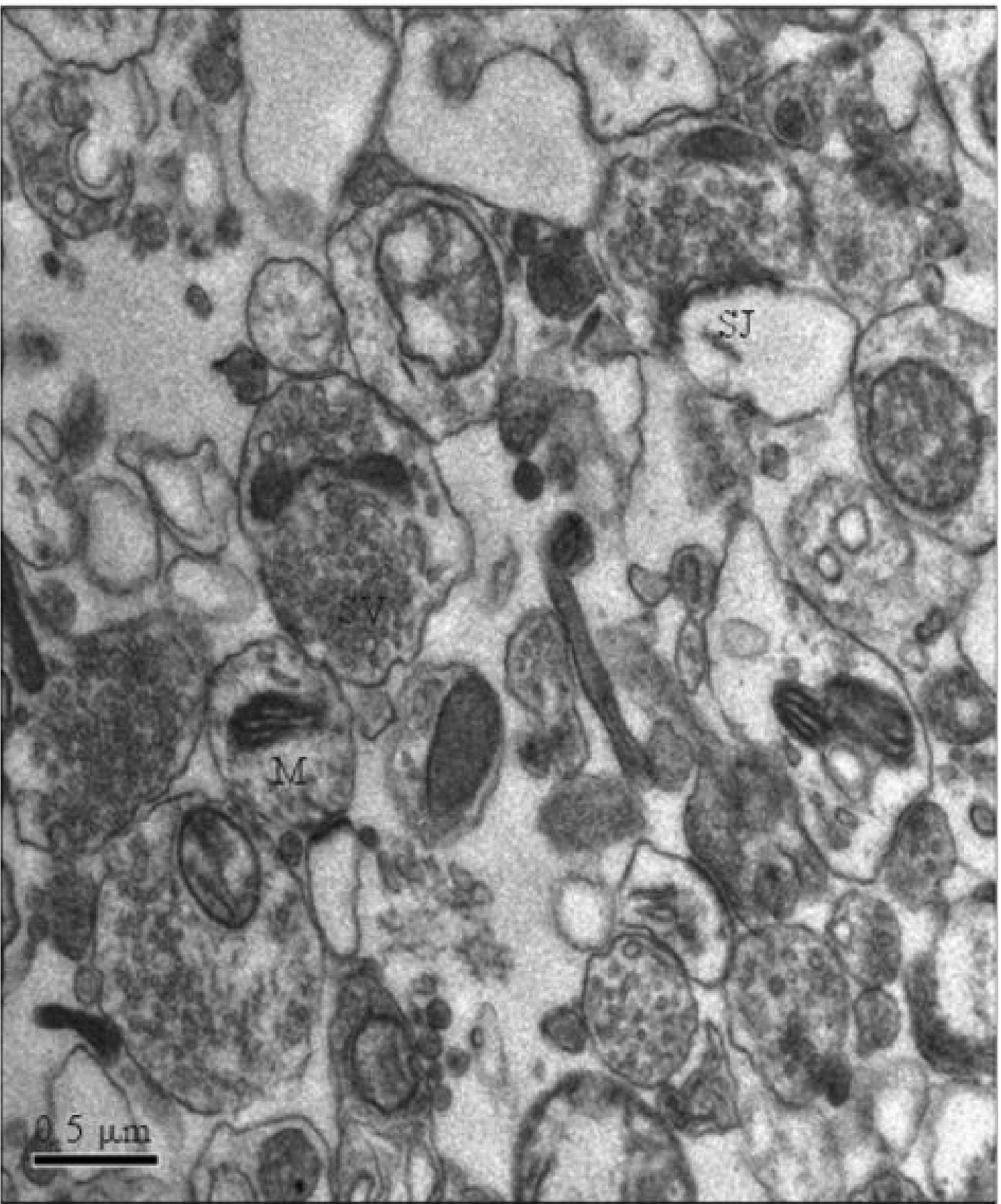

Figure 2.

Electron micrograph of a synaptosome fraction isolated from mouse brain by Ficoll densitygradient centrifugation. SV, synaptic vesicles; $\mathrm{M}$, mitochondria; SJ, synaptic junction with attached PSyD. Bar $=0.5 \mathrm{~mm}$. (From: Schrimpf et al. 2005) 


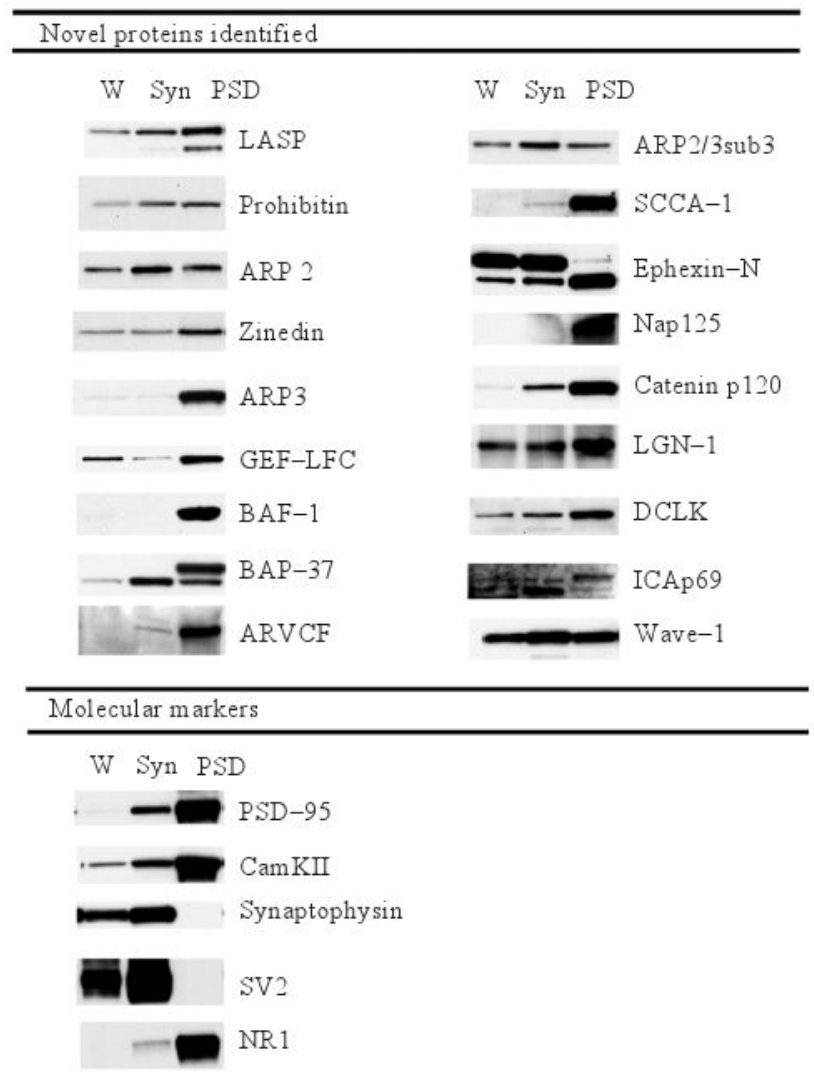

Figure 3.

Western blots of several novel members of the PSD. Ten to $20 \mu \mathrm{g}$ of PSD protein were separated by SDS-PAGE and blotted using a variety of antibodies. W, whole brain extract; Syn, synaptosomal fraction; and PSD, postsynaptic density. Known molecular markers were also blotted to demonstrate the purity of the fractions used for MS/MS. ARP, actin-related protein; BAF-1, barrier to autointegration 1; BAP-37, B cell receptor-associated protein; ARVCF, armadillo-repeat-velo-cardio-facial syndrome protein; ARP2/3sub5, actin-related protein complex 2/3 subunit 5; SCCA-1, squamous cell carcinoma antigen-1, serpin3a; NAP-125, NCK-associated protein 1; LGN-1, leucine-rich repeat LGI family, member 1; DCLK, doublecortin-like kinase/mKIAA0369; ICAp69, islet cell autoantigen protein p69. (From: Jordan et al. 2004). 


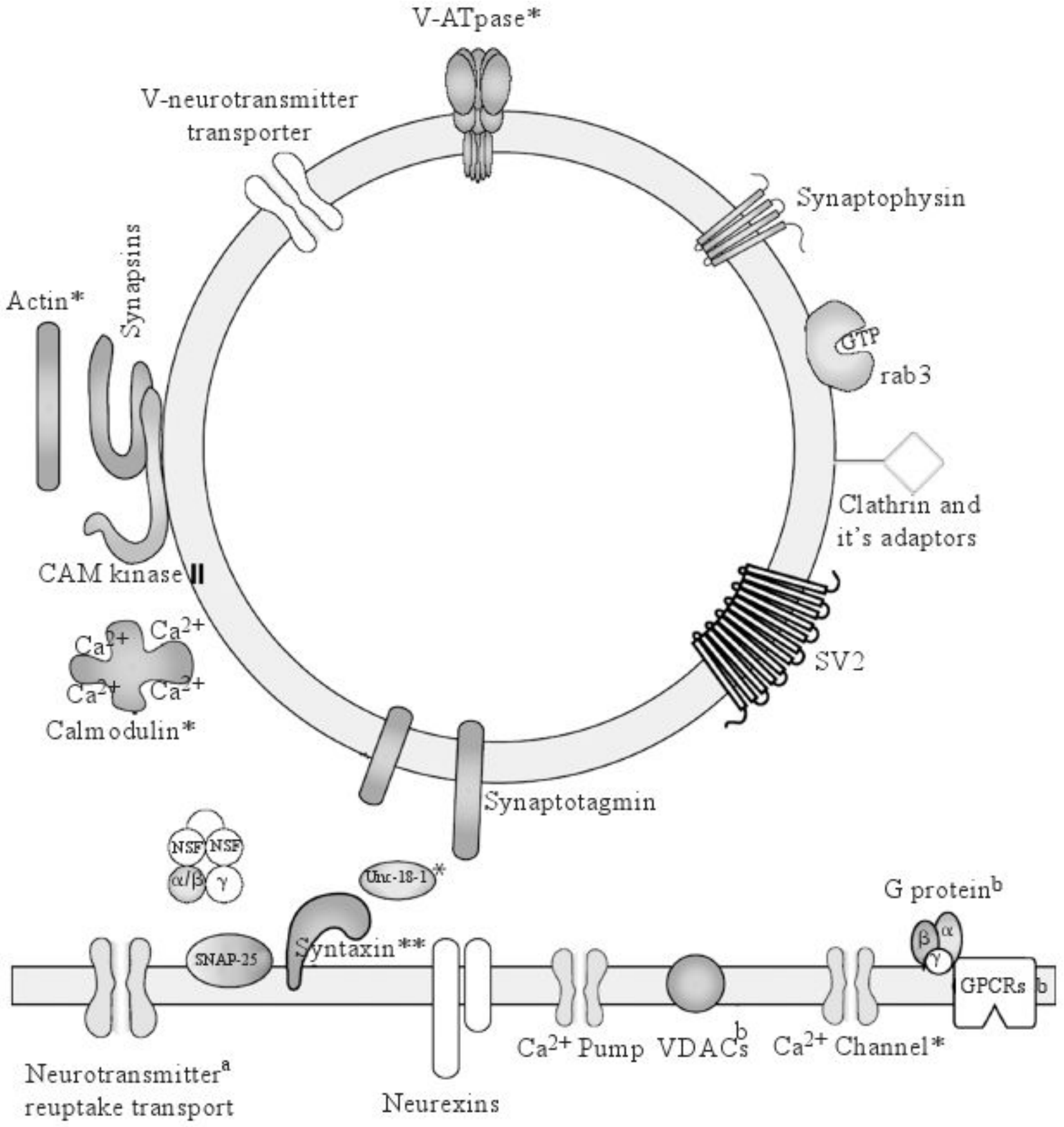

Figure 4.

Diagrammatic illustration of the major pre- and postsynaptic proteins identified by 2-DE/MS and/or shotgun proteomics, and normally expected as constituent in synaptosomal preparations. Blue: the proteins that were identified by 2-DE/MS; Yellow: the proteins that were identified by shotgun proteomics; Green: the proteins that were identified by both 2-DE/ MS and shotgun proteomics; and Blank (white): those major constituents expected but not identified. *proteins were identified by shotgun proteomics only after the PTM analysis; **proteins were identified as a complex with other proteins by 2-DE/MS; ${ }^{\mathrm{a}} \mathrm{EAA} 1, \mathrm{EAA} 2$, and GABA transporter. ${ }^{b}$ post-synaptic proteins. (From: Witzmann et al. 2005) 
(A)

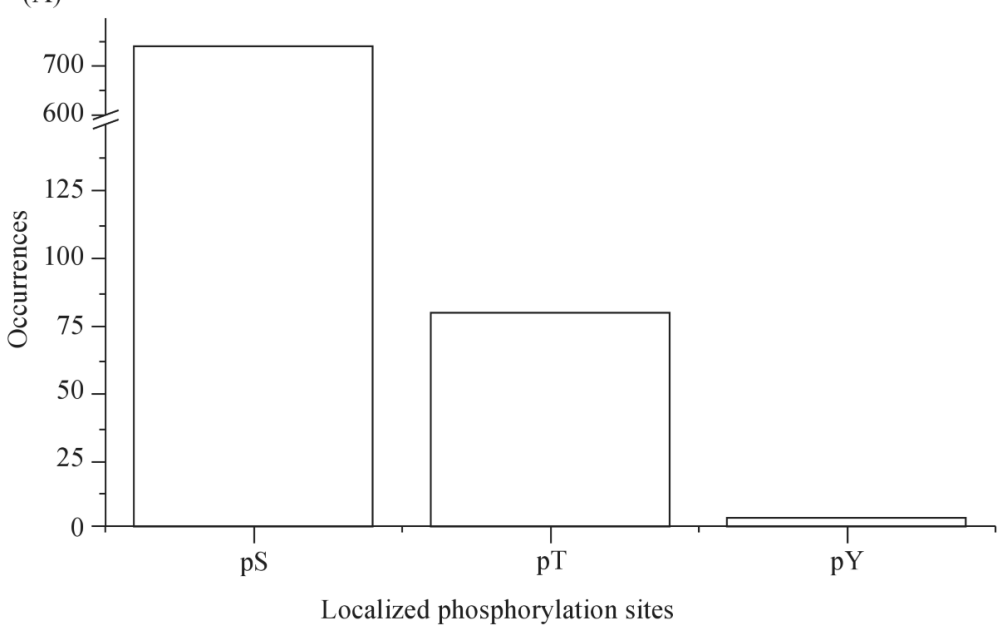

(B)

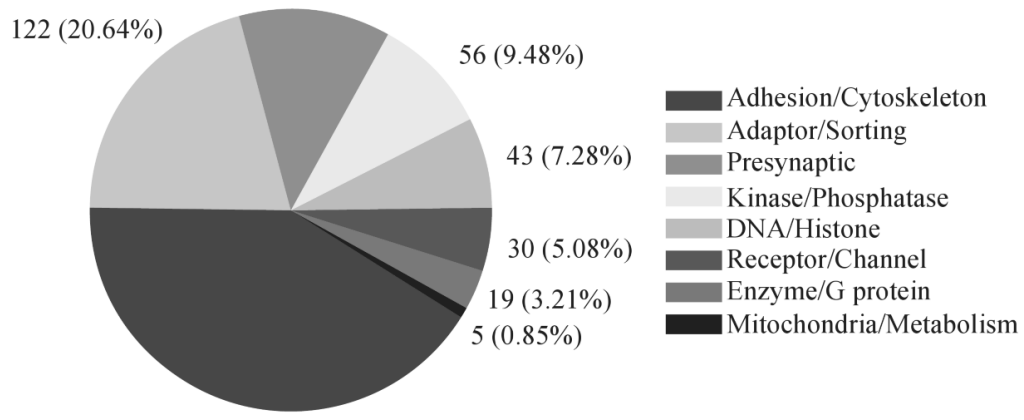

$244(41.29 \%)$

Figure 5.

(A). the relative distribution of phosphoserines $(\mathrm{pS})$, phosphothreonines $(\mathrm{pT})$, and phosphotyrosines (pY). The majority of identified sites were phosphoserines (739) followed by phosphothreonines (79) and lastly phosphotyrosines (three). (B). the number of unique phosphorylation sites as a function of the protein functional class. Proteins involved in adhesion/cytoskeleton represent the most prevalent class followed by proteins involved in adaptor/sorting functions. (From: Trinidad et al. 2006) 
Table 1

Protein fractions derived from synapses and the proteomic methods used to study them

\begin{tabular}{|c|c|c|c|}
\hline Synaptic Fraction Source & Proteomic Platform & Proteome Analyzed & Reference \\
\hline \multicolumn{4}{|l|}{ Synaptosomes } \\
\hline Rat Brain & 1D-PAGE, 2-DE; LC-MS/MS; Western blot & O-GlcNAc modified proteins & (Cole et al. 2001) \\
\hline Human Cortex & IMAC; LC-MS/MS & Phosphoproteome & (DeGiorgis et al. 2005) \\
\hline Gerbil cortex & $\begin{array}{l}\text { 2-DE; MALDI-TOF; LC-MS/MS; Western } \\
\text { blot }\end{array}$ & Redox proteome & $\begin{array}{l}\text { (Boyd-Kimball et al. } \\
\text { 2005) }\end{array}$ \\
\hline Mouse Brain & IMAC; LC-MS/MS & Phosphoproteome & (Collins et al. 2005a \& b) \\
\hline Mouse Brain & ICAT; LC-MS/MS & Protein ID & (Schrimpf et al. 2005) \\
\hline Rat forebrain & $\begin{array}{l}\text { Affinity enrichment; 2-DE; MALDI-TOF; } \\
\text { LC-MS/MS }\end{array}$ & Protein ID, shotgun, and PTMs & (Witzmann et al. 2005) \\
\hline \multicolumn{4}{|l|}{ Synaptic Vesicles } \\
\hline Rat brain & 2-DE, 16-BAC/SDS-PAGE; LC-MS/MS & Protein ID & (Coughenour et al. 2004) \\
\hline Rat brain & 16-BAC/SDS-PAGE; MALDI-TOF & Protein ID & (Morciano et al. 2005) \\
\hline \multicolumn{4}{|l|}{ Synaptic membranes } \\
\hline Rat forebrain & $\begin{array}{l}\text { SDS-PAGE, 2-DE, SCX, HPLC; MALDI- } \\
\text { TOF, LC-MS/MS }\end{array}$ & Protein ID & (Stevens et al. 2003) \\
\hline Rat forebrain & 2-DE; LC-MS/MS & Protein ID & (Li et al. 2004) \\
\hline Rat brain & ICAT; LC-MS/MS & Differential expression & (Prokai et al. 2005) \\
\hline $\begin{array}{l}\text { Rat brain (SV \& presyn. } \\
\text { mem.) }\end{array}$ & 6-BAC/SDS-PAGE; MALDI-TOF & Protein ID & (Morciano et al. 2005) \\
\hline \multicolumn{4}{|l|}{ Postsynaptic Density (PSD) } \\
\hline Rat forebrain & SDS-PAGE; MALDI-TOF & Protein ID & (Walikonis et al. 2000) \\
\hline Mouse forebrain & 2-DE; LC-MS/MS & $\begin{array}{l}\text { Protein ID and differential } \\
\text { expression }\end{array}$ & (Satoh et al. 2002) \\
\hline Rat forebrain & 2-DE; MALDI-TOF; ICAT; LC-MS/MS & Protein ID & (Li et al. 2004) \\
\hline Rat forebrain & MudPIT & Protein ID & (Yoshimura et al. 2004) \\
\hline Rat \& mouse brain & SDS-PAGE; LC-MS/MS; Western blot & Protein ID & (Jordan et al. 2004) \\
\hline Rat forebrain & SDS-PAGE; LC-MS/MS & $\begin{array}{l}\text { Protein ID and semi- } \\
\text { quantification }\end{array}$ & (Peng et al. 2004) \\
\hline Rat brain & $\begin{array}{l}\text { ICAT, SCX; LC-MS/MS; Immunoelectron } \\
\text { microscopy }\end{array}$ & Protein ID and quantification & (Li et al. 2005) \\
\hline Rat cortex & MudPIT & Protein ID & (Phillips et al. 2005) \\
\hline Rat forebrain \& cerebellum & ICAT; LC-MS/MS & $\begin{array}{l}\text { Relative and absolute } \\
\text { quantification }\end{array}$ & (Cheng et al. 2006) \\
\hline Mouse brain & $\begin{array}{l}\text { IP, affinity purification; 2-DE; LC-MS/MS; } \\
\text { Western blot }\end{array}$ & Protein ID & (Collins et al. 2005b) \\
\hline Rat hippocampus & MUDPIT & Protein ID & (Dosemeci et al. 2006) \\
\hline Mouse brain & SCX, IMAC; LC-MS/MS; Western blot & Phosphoproteome & (Trinidad et al. 2006) \\
\hline Mouse brain & $\begin{array}{l}\text { lectin weak affinity chromatography; O- } \\
\text { GlcNAc LC-MS/MS; MALDI-TOF }\end{array}$ & modified proteins & (Vosseller et al. 2006) \\
\hline \multicolumn{4}{|l|}{$\begin{array}{l}\text { Presynaptic Particle Fraction } \\
\text { (PPF) }\end{array}$} \\
\hline Rat cortex & MudPIT & Protein ID & (Phillips et al. 2005) \\
\hline
\end{tabular}

